\title{
Chemical wave propagation in Hele-Shaw cells and porous media
}

\author{
Desiderio A. Vasquez \\ Department of Physics, Indiana University-Purdue University Fort Wayne, Fort Wayne, \\ Indiana 46805-1499 \\ Joseph W. Wilder \\ Department of Mathematics, West Virginia University, P.O. Box 6310, Morgantown, \\ West Virginia 26506-6310 \\ Boyd F. Edwards \\ Department of Physics, West Virginia University, P.O. Box 6315, Morgantown, West Virginia 26506-6315
}

(Received 30 October 1995; accepted 21 March 1996)

\begin{abstract}
Chemical waves induce density gradients in fluids which may lead to convection. This paper studies the convective effects on chemical waves propagating in porous media or in fluids confined between two parallel vertical walls. Chemical waves in the iodate-arsenous acid system are modeled with a one variable reaction-diffusion equation. The fluid flow is modeled using Darcy's law. A linear stability analysis on convectionless fronts shows a transition to convection. The full nonlinear equations describing the convective front are solved numerically on a vertical slab. Convective fronts propagate faster than convectionless fronts. Near the onset of convection, the fronts are raised on one side of the slab and lowered on the opposite side. Away from the onset, the fronts are raised at the middle of the slab with the opposing sides at the same height. (C) 1996 American Institute of Physics. [S0021-9606(96)01524-3]
\end{abstract}

\section{INTRODUCTION}

Chemical waves propagate in liquids and polyacrilamide gels due to the interaction between molecular diffusion and chemical autocatalysis. ${ }^{1}$ In experiments in chemical waves, convection arises from density gradients induced either by compositional gradients or by the exothermicity of the reaction. ${ }^{2}$ The theory of chemical wave propagation based on reaction-diffusion models neglects the effects due to convection. In experiments in the iodate-arsenous acid reaction, convection can increase the chemical wave speed by up to a factor of $4,{ }^{3}$ where as in other reactions such as the iodatenitric acid reaction the speed can increase by a factor of $50 .{ }^{4}$ Convection also plays an important role in determining the shape of the chemical wave. Waves propagating upward in vertical capillary tubes can be flat, axisymmetric, or nonaxisymmetric depending on the diameter of the tube. ${ }^{5}$ Experimental observations of convection in chemical waves have taken place in viscous fluids. In petri dishes and capillary tubes the fluid motion can be better described using the full Navier-Stokes equations. For convection in porous media, such as polyacrilamide gels, the fluid motion obeys Darcy's law. This model can also be applied to fluids confined between two vertical walls with a small separation as in a Hele-Shaw cell. ${ }^{6}$ In this paper we analyze convection in chemical waves under Darcy's law and compare it with Navier-Stokes flows. We find significant differences (quantitative and qualitative). These differences are important for future experiments.

In this paper we present a theory for the transition to convection for ascending chemical waves propagating upward. We concentrate on reaction fronts in the iodatearsenous acid reaction with arsenous acid in stoichiometric excess, which can be modeled with a one-variable reaction- diffusion equation. Although this reaction is exothermic, the heat equation can be neglected because the length scale of thermal gradients is much larger than the length scale for the convection produced. This corresponds to the limit of infinite thermal diffusivity. We carry out a linear stability analysis for small plane wave perturbations to the convectionless front. We compare the results of the analysis of the reactiondiffusion equation to the results obtained using a thin front eikonal approximation. We numerically solve the full nonlinear reaction-diffusion equations coupled to Darcy's law. We calculate the increase of speed and change in shape for convective waves in vertical slabs.

\section{EQUATIONS OF MOTION}

In this work we compare two models for the propagation of the chemical front, one based on a one-variable reaction diffusion equation and the other based on a thin front approximation. These equations are coupled to Darcy's law which describes fluid flow in porous media. The thin front approximation considers the chemical wave as a surface that separates reacted from unreacted fluid. This approximation consists of an eikonal relation ${ }^{7}$ involving the normal velocity of the chemical front $(v)$, the flat front velocity $\left(v_{0}\right)$, its curvature $(\kappa)$ and the diffusion coefficient $(D)$

$$
v=v_{0}+D \kappa+\hat{n} \cdot \mathbf{V} .
$$

The fluid velocity at the front $(\mathbf{V})$ in the direction parallel to the unit vector $\hat{n}$ normal to the front adds to the normal speed of the chemical front. We choose a realistic one-variable reaction-diffusion model $^{8}$ developed for convectionless fronts in the iodate-arsenous acid reaction with arsenous acid in stoichiometric excess 


$$
\frac{\partial c}{\partial t}=D \nabla^{2} c-\alpha c\left(c-c_{2}\right)\left(c-c_{3}\right) \equiv D \nabla^{2} c+f(c) .
$$

Here, $D$ is the molecular diffusivity, $c$ is the iodide concentration $\left(\left[\mathrm{I}^{-}\right]\right), c_{2}$ is the initial concentration of iodate $\left(\left[\mathrm{IO}_{3}^{-}\right]\right)$, $c_{3}$ is the ratio of two reaction rate constants: $c_{3}=-k_{a} / k_{b}$ and $\alpha=k_{b}\left[\mathrm{H}^{+}\right]^{2}$. The value of the reaction rate constants are $k_{a}=4.50 \times 10^{3} \mathrm{M}^{-3} \mathrm{~s}^{-1}$ and $k_{b}=3.45 \times 10^{8} \mathrm{M}^{-4} \mathrm{~s}^{-1}$. The value of $k_{b}$ ensures that the theoretical wave speed $v_{0}$ agrees with the speed measured in experiments. ${ }^{9}$ This equation is satisfied by a propagating front solution with constant speed

$$
c(\mathbf{x}, t)=\frac{c_{2}}{1+B e^{k\left(z-v_{0} t\right)}}
$$

Here, $B$ is an arbitrary constant that specifies the initial position of the front. The direction of propagation is the vertical $z$ direction. The front velocity $v_{0}$ and the parameter $k$ are related to the chemical parameters by

$$
v_{0}=\left(D k_{b} / 2\right)^{1 / 2}\left[\mathrm{H}^{+}\right]\left[\mathrm{IO}_{3}^{-}\right]+\left(2 D / k_{b}\right)^{1 / 2} k_{a}\left[\mathrm{H}^{+}\right]
$$

and

$$
k=\left(k_{b} / 2 D\right)^{1 / 2}\left[\mathrm{H}^{+}\right]\left[\mathrm{IO}_{3}^{-}\right] .
$$

We shall use typical experimental values ${ }^{7}$ of the concentrations: $\left[\mathrm{H}^{+}\right]=10^{-2} \mathrm{M}$ and $\left[\mathrm{IO}_{3}^{-}\right]=5 \times 10^{3} \mathrm{M}$. In the presence of convection the reaction-diffusion equation is coupled to Darcy's law and the continuity equation describing the flow in porous media

$$
\begin{aligned}
& \mathbf{V}=-\frac{K}{\mu}(\nabla P+\rho g \hat{z}), \\
& \boldsymbol{\nabla} \cdot \mathbf{V}=0, \\
& \frac{\partial c}{\partial t}+(\mathbf{V} \cdot \boldsymbol{\nabla}) \mathbf{V}=D \nabla^{2} c+f(c) .
\end{aligned}
$$

Here, $K$ is coefficient of permeability of the medium, $g=980$ $\mathrm{cm} / \mathrm{s}^{2}$ the acceleration of gravity along the vertical $\hat{z}$ direction, $\rho$ the fluid density, $\mu$ is the coefficient of viscosity which is related to the kinematic viscosity by $\nu=\mu / \rho$. The fluid density is set to vary linearly with the iodide concentration $c$

$$
\rho=\rho_{0}\left[1-\beta\left(c-c_{2}\right)\right],
$$

with $\beta=1.7 \times 10^{-2} \mathrm{M}^{-1}$ the coefficient of linear expansion for the iodide-arsenous acid reaction under the conditions previously described. It has been shown that the flow for a viscous liquid confined between two walls can be modeled with Darcy's law if the separation between the walls $(a)$ is small enough. In this approximation the coefficient of permeability is replaced by $K=a^{2} / 12$.

We solve the equations for a laterally unbounded front and for a front confined in the horizontal direction. The equations are two-dimensional in the Cartesian $x-z$ plane, with $z$ being the vertical direction. In this system, we can eliminate the reduced pressure and satisfy the continuity equation identically by defining the stream function $\psi$ with

$$
V_{x}=\frac{\partial \psi}{\partial z}, \quad V_{z}=-\frac{\partial \psi}{\partial x} .
$$

This allows us to rewrite the equations in dimensionless form as

$$
\begin{aligned}
& \frac{\partial c}{\partial t}=\frac{\partial(\psi, c)}{\partial(x, z)}+\nabla^{2} c-\alpha^{\prime} c(c-1)\left(c-c_{3} / c_{2}\right), \\
& \nabla^{2} \psi=-\operatorname{Ra} \frac{\partial c}{\partial x} .
\end{aligned}
$$

Here, we define

$$
\frac{\partial\left(f_{1}, f_{2}\right)}{\partial(x, z)}=\frac{\partial f_{1}}{\partial x} \frac{\partial f_{2}}{\partial z}-\frac{\partial f_{2}}{\partial x} \frac{\partial f_{1}}{\partial z} .
$$

The variables are scaled such that $c$ is measured in terms of $c_{2}, \psi$ is measured in terms of $D, \alpha^{\prime}$ is equal to $\alpha L^{2} c_{2}^{3} / D^{2}$, and $\mathrm{Ra}$ is the Rayleigh number

$$
\mathrm{Ra}=\frac{K \delta g L}{\nu D} .
$$

Here, $L$ is a convenient length scale. In a Hele-Shaw cell, $L=a$, the wall separation, whereas $L=\sqrt{12 K}$ for porous media. Time is measured in units of $L^{2} / \nu$. The Rayleigh number for the Hele-Shaw cell is therefore given by

$$
\mathrm{Ra}=\frac{a^{3}}{12} \frac{\delta g}{\nu D}=\frac{\varsigma}{12},
$$

which involves the dimensionless number $\mathfrak{s}$ defined for the bounded front in a viscous fluid. ${ }^{10}$

\section{LINEAR STABILITY ANALYSIS}

The zeroth order convectionless solution is given by Eq. (3). A linear stability analysis is conducted by introducing perturbations of the form

$$
\psi=\psi^{(0)}+\psi^{(1)}
$$

and

$$
c=c^{(0)}+c^{(1)} .
$$

Substituting these expressions into Eqs. (11) and (12) and keeping only the terms linear in the perturbations we find in a frame comoving with the front

$$
\frac{\partial c^{(1)}}{\partial t}=\frac{\partial \psi^{(1)}}{\partial z} \frac{d c^{(0)}}{d z}+\nabla^{2} c^{(1)}+\left.v_{0} \frac{d c^{(1)}}{d z} \frac{d f}{d z}\right|_{c^{(0)}}
$$

and

$$
\nabla^{2} \psi^{(1)}=-\operatorname{Ra} \frac{\partial c^{(1)}}{\partial x} .
$$

For the laterally unbounded problem, we study plane wave perturbations of the form

$$
c^{(1)}=c(z) e^{\sigma t} \cos (q x)
$$

and

$$
\psi^{(1)}=\psi(z) e^{\sigma t} \sin (q x) .
$$




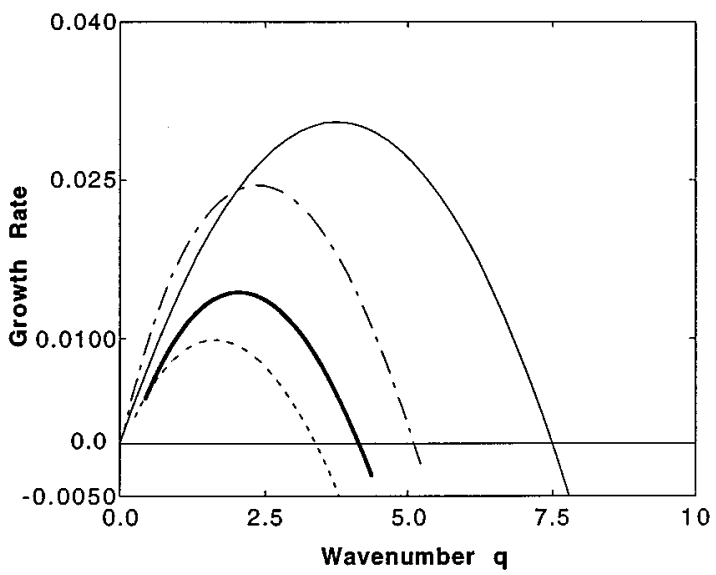

FIG. 1. The growth rate as a function of wave number for perturbations to the flat convectionless front. The bold solid line represents a value of $\mathrm{Ra}$ $=15$, the dotted line is for $\mathrm{Ra}=10$ and the dashed-dotted line represents $\mathrm{Ra}=20$. These three values correspond to a linear stability analysis of the reaction-diffusion model. The $\mathrm{Ra}=15$ curve is compared to the one generated with the eikonal relation (solid line). The results are significantly different although the value of the Rayleigh number is the same. The units are the dimensionless units described in the text.

With these substitutions, the equations become a set of ordinary differential equations in the $z$ direction that define the growth rate $\sigma$ for every value of the wave number $q$. These equations can be solved numerically using a shooting method. The method consists of starting one solution far above the front, and another far below the front, using the differential equations to continue each solution to the front. In order to start the shooting method, the boundary conditions are supplemented with some additional trial conditions. The trial conditions are chosen to cause the two solutions to match at the front. This method was used to calculate the growth rate as a function of wave number, for the iodatearsenous acid equation in a viscous fluid. The method is similar to the one described in Ref. 9 with the added advantage that Darcy's law is of lower order than the NavierStokes equation.

The results of the linear stability analysis are shown in Fig. 1 for three values of the Rayleigh number $\mathrm{Ra}=10,15$, and 20. For these cases the growth rate $(\sigma)$ is positive for wave numbers $(q)$ close to zero, and the growth rate goes to zero as the wave number goes to zero. This means that flat fronts are always unstable to perturbations of large wavelength $(\lambda=2 \pi / q)$. In Fig. 1, we notice that the growth rate increases with $q$ until it reaches a maximum. After this the growth rate decreases and eventually becomes negative for large wave numbers. The wave number for marginal stability $\left(q_{c}\right)$ is reached when $\sigma=0$, and separates the wave numbers of stable and unstable perturbations. For all values $q<q_{c}$ the perturbations are unstable while for $q>q_{c}$ the perturbations are stable. Consequently, perturbations of small enough wavelength are stable. We find that $q_{c}$ increases as the Rayleigh number increases. For $\mathrm{Ra}=10,15$, and 20 , the values of $q_{c}$ are 3.39, 4.00, and 5.11, respectively. This increases the range for wave numbers with positive growth rate, and

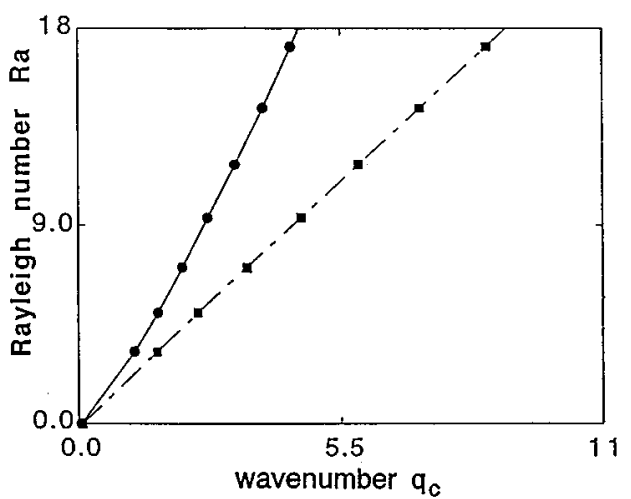

FIG. 2. The critical wave number for marginal stability for different Rayleigh numbers. The dotted line represents the values obtained with an eikonal relation while the solid line represents the results of the reactiondiffusion model. The units are the dimensionless units described in the text.

consequently the front will be more unstable for large Rayleigh numbers. The Rayleigh number can be changed experimentally by controlling the width of the Hele-Shaw cell. These results show that a front propagating in a Hele-Shaw cell with a small gap is more stable than one propagating in a cell with a larger gap.

We shall now compare the results for the linear stability analysis using the reaction-diffusion equation with those from using the eikonal relation. The eikonal relation gives an analytic expression for the dispersion relation ${ }^{10}$

$$
\sigma=\frac{D}{\nu} q\left(\frac{\mathrm{Ra}}{2}-q\right)
$$

having two zeros, one with $q=0$ and the other with $q=\mathrm{Ra} / 2$. While the reaction-diffusion equation also provides the $q=0$ solution, the critical wave number $q_{c}$ is significantly different from the one obtained with the eikonal relation. For example, for $\mathrm{Ra}=15$ the critical wave number $q_{c}$ is equal to 4.00 for the reaction-diffusion equation and 7.5 for the eikonal relation. In Fig. 2 we compare the results for the critical wave number as a function of the Rayleigh number using the reaction-diffusion equation and the eikonal relation. While both results are reasonably close for small Rayleigh numbers, the results separate as the Rayleigh increase. This difference between the eikonal relation and the reactiondiffusion equation contrasts the good agreement between both models for fronts propagating in viscous fluids. Previous work has shown ${ }^{9}$ that in viscous fluids the difference between the critical wave numbers resulting from these models is on the order of $5 \%$.

The growth rate as a function of wave number shows a maximum growth rate for all cases (Fig. 1). This maximum growth rate lies at wave numbers $q=1.63,1.90$, and 2.36 for $\mathrm{Ra}=10,15$, and 20 , respectively. The maximum growth rate increases as the Rayleigh number is increased. The maximum growth rate for $\mathrm{Ra}=15$ is equal to 0.014 , which is different from the maximum growth rate given the eikonal relation $(\sigma=0.030)$. The wave number for the maximum growth rate in the eikonal relation is equal to one-fourth of 
the Rayleigh number, which is exactly half the critical wave number $q_{c}$. This relation does not hold for the reactiondiffusion equation, although it is close for the Rayleigh numbers studied. For example, the maximum growth rate for $\mathrm{Ra}=15$ lies at 1.90 and half the critical wave number is equal to 2.00. These differences in critical wave numbers and growth rate lead us to conclude than the eikonal relation provides a less stable front that the reaction-diffusion equation. For small Rayleigh numbers there is reasonable agreement between the two models. However, as the Rayleigh number increases, the difference between them becomes more significant.

\section{NONLINEAR SOLUTIONS}

We investigate the two-dimensional behavior of the convective chemical front by solving numerically the full set of nonlinear equations (10) and (11). These equations are supplemented with no flow boundary conditions for the chemical concentrations and no normal fluid velocity at the walls. The boundary conditions for the fluid velocity translates into zero tangential derivatives for the stream function. We choose the two-dimensional rectangular box as our domain since it is the simplest geometry that allows convective fluid motion. We discretize the spatial domain with a rectangular mesh. The spatial derivatives are evaluated using second order finite differences. The horizontal size of the domain is varied while the vertical size is fixed. The number of grid points is increased to reflect an increase in the horizontal size of the domain. The time evolution is carried out using a simple Euler technique with a small time step. Other techniques, such as an alternate direction implicit (ADI) technique did not improve the computational time. The Poisson equation (11) was solved using the subroutine GENBUN of the FISHPACK package ${ }^{11}$ which makes use of cyclic reduction technique. This proved more efficient and more accurate than using the successive over relaxation method (SOR). The front is initiated by filling a region close to the bottom of the domain with concentrations of reacted fluid, and with unreacted fluid elsewhere. Small random initial perturbations were added to test the effects of the initial conditions. As the front propagates vertically in this bounded rectangular domain it will eventually collide with the upper boundaries. To study the long time behavior of the front would require an infinite vertical domain. Instead, we shift the front downward when it nears the upper boundary. We add unreacted convectionless fluid near the upper boundary and discard the reacted convectionless fluid near the lower boundary as we shift the front. This procedure is justified as long as the shift begins and ends with the front far away from the vertical boundaries, since all of the velocity and concentration gradients are near the front. We performed tests with larger vertical domains; the solutions remained unchanged.

We compare our results of the nonlinear calculations with the results of the linear stability analysis near the onset of convection. In Fig. 3 we show the increase of speed near the onset of convection as we vary the horizontal size of our rectangular domain. The results are compared to the results

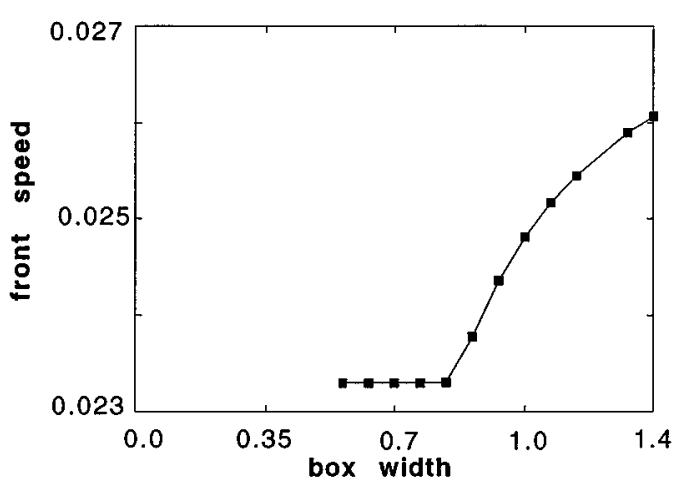

FIG. 3. The chemical wave velocity as a function of box width. The value for the coefficient of permeability is set to $0.043 \mathrm{~mm}^{2}$. The unit of length corresponds to a Hele-Shaw cell separation of 0.72 . For widths below 0.76 there is no convection present and all the waves have the same speed. For widths above $0.83 \mathrm{~mm}$ convection sets in and increases the wave speed. The units are the dimensionless units described in the text with $L=0.72 \mathrm{~mm}$.

of the linear stability analysis. The boundary conditions for our bounded domain can be related to the unbounded calculations by choosing the corresponding wave number $q=\pi / L_{x}$, where $L_{x}$ corresponds to the width of the box. With this choice of wave number the linear stability analysis satisfies the same boundary conditions as the bounded systems. This is allowed since Darcy's law (being of second order) requires free boundary conditions for the fluid velocity, that is zero normal velocity and free tangential velocity at the boundary. In the case of a viscous fluid, the NavierStokes equation would required two boundary conditions for the velocity field corresponding to zero fluid velocity at the walls of the rectangular box and, consequently, a direct comparison between the unbounded and bounded system would not be possible. The linear stability analysis developed in the previous section showed that perturbations of wavelength greater than a critical wavelength have a negative growth rate and consequently will die out. The boundaries on the nonlinear simulations act as a wavelength selector since the only perturbations that can be introduced will have a wavelength smaller than the width of the box. This fact, together with free boundary conditions, allows for a direct comparison of the linear stability analysis with the full numerical calculations.

The nonlinear calculations start with a flat interface between reacted and unreacted fluid subject to small random perturbations near the front. The stream function is set to zero everywhere. For our nonlinear calculations we chose the value of the coefficient of permeability to be $0.043 \mathrm{~mm}^{2}$ which corresponds to a wall separation of $a=0.72 \mathrm{~mm}$ in a Hele-Shaw cell, which becomes our unit of length. The value of the Rayleigh number corresponds to 14.4. The total vertical length of our domain of integration is 10.4 (dimensionless units) using 150 grid points to discretize the vertical direction. We varied the horizontal width but kept the horizontal distance between mesh points close to $7 \times 10^{-1}$. As we show in Fig. 3, for a domain of width less than 0.76 , the initial state evolves into a convectionless front propagating 


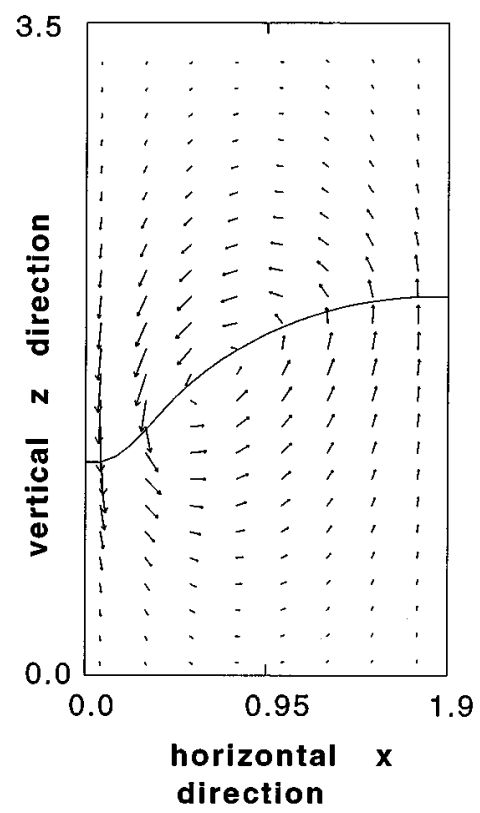

FIG. 4. Velocity profile for a convective chemical wave in a box of width 1.9. The solid line separates reacted from unreacted fluid. The front is curved due to a single convective roll. The units are the dimensionless units described in the text with $L=0.72 \mathrm{~mm}$.

upward. All fronts in boxes of smaller width are horizontally flat with the same velocity as the purely reaction-diffusion front. For widths larger than 0.83 we found that the front evolves into a curved front with one side of the front higher than the opposing side, moving vertically with constant speed (Fig. 4). This change in shape is due to the convective fluid motion that consists of a single roll, with fluid rising on one side of the box and falling on the opposite side. The presence of convection increases the speed of the front as it enhances the mixing of the chemicals. Convection becomes stronger as the width of the tube is further increased leading to a larger front speed. The numerical simulations established that the critical box width that allows convective fronts should be between 0.76 and 0.83 . These results are in excellent agreement with the linear stability analysis based on the reaction diffusion equation that gave a critical width of 0.81 . The calculations based on the eikonal relation gives a critical width of 0.44 which is in disagreement with the nonlinear results. This reaffirms the fact that the eikonal relation is not a very good approximation when the fluid motion obeys Darcy's law.

As the width of the box is increased well above the onset of convection, a new type of front develops from the standard initial conditions (flat front plus small random perturbations). This front is higher on a vertical line running through the center of the box and is lower near the walls (Fig. 5). This front is symmetric with respect to a reflection about this vertical line. This symmetry is caused by two counterrotating convective rolls that consist of fluid rising near the middle and falling close to the vertical walls. Once we obtained a symmetric front, we used it as the initial condition for a front in a box of slightly larger or smaller width. The speed of the

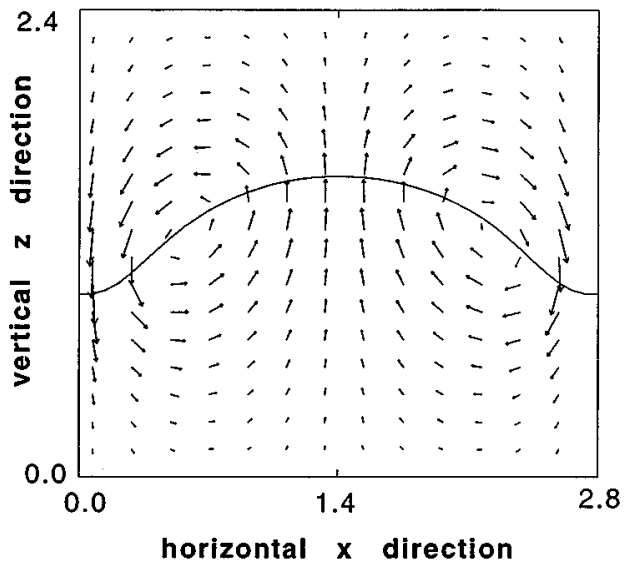

FIG. 5. Velocity profile for a convective chemical wave in a box of width 2.8. The solid line separates reacted from unreacted fluid. The front curvature is axisymmetric due to two identical, counterrotating convective rolls. The units are the dimensionless units described in the text with $L=0.72 \mathrm{~mm}$.

symmetric front increases as the width increases. We applied the same procedure for the nonsymmetric fronts that developed near the onset of convection. Our results are summarized in Fig. 6 where we show the speeds of both types of fronts as a function of the width. Near the onset of convection the only fronts allowed correspond to the nonsymmetric fronts with a single convective roll. However, Fig. 6 shows a region of bistability where we can find either a nonsymmetric or a symmetric front. In this region, the symmetric fronts are slower than the nonsymmetric fronts for widths smaller than 3.2, and are faster for larger widths. Our numerical calculations did not produce symmetric fronts below $0.18 \mathrm{~mm}$ nor nonsymmetric fronts above $3.4 \mathrm{~mm}$. This may indicate that the fronts become unstable near those widths, although a complete treatment of this question will require a stability analysis of the convective front beyond the scope of the present work. All of the steady fronts consist of either a single convective roll or two identical counterrotating rolls. In some cases, a transient state consisting of a stronger and a

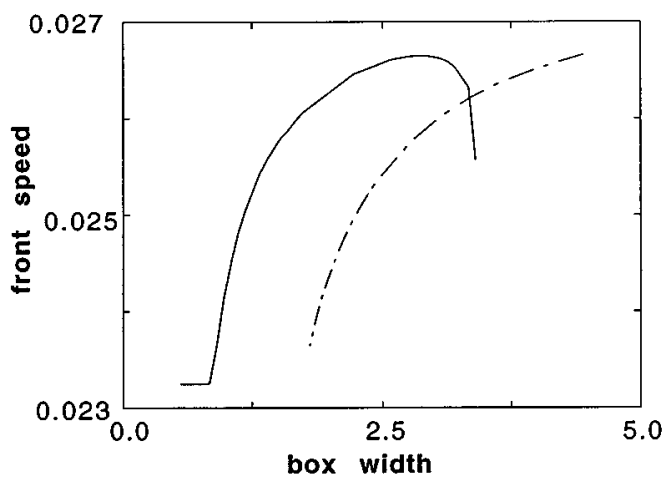

FIG. 6. The chemical wave velocity as a function of box width. The solid line represents a front with only one convective roll. The broken line represents an axisymmetric front with two convective rolls. There are width values where both kinds of fronts can be formed. The units are the dimensionless units described in the text with $L=0.72 \mathrm{~mm}$. 
weaker roll was observed, but it inevitably evolved onto either of the two previous cases. This is in stark contrast with simulations with the Navier-Stokes equations, where the steady state correspond to a "mixed" state of symmetric and nonsymmetric fronts with two different convective rolls.

\section{DISCUSSION}

The results of the linear stability analysis show that a laterally unbounded front propagating upward will always be unstable for perturbations of long enough wavelength. However, perturbations with wavelengths smaller than a critical wavelength will be stable. This qualitative result is valid for both the reaction-diffusion and the eikonal relation models, although they disagree in the exact value of the cutoff wavelength. The reaction-diffusion model predicts a front that is more stable than the eikonal relation. This result was tested with a numerical solution of the full nonlinear reactiondiffusion-convection equations. The boundaries introduced in this calculation served as wavelength filters since large wavelength perturbations cannot fit between them. A previous study of the three dimensional effects on a Hele-Shaw cell showed that approximating the viscous flow with Darcy's law is valid only when the gap between the walls is small. ${ }^{10}$ This study utilized the eikonal relation for the front propagation. The present work shows that another important factor to take into account is the thickness of the front. The results of the reaction-diffusion equation differ significantly from the ones obtained with the eikonal relation when the flow is represented with Darcy's law. The results provided by the reaction-diffusion model should better describe the behavior of the chemical front.

Convection in chemical waves has been observed in different chemical reactions. ${ }^{12,13}$ Experiments showed symmetric and nonsymmetric fronts for chemical waves propagating inside vertical capillary tubes. ${ }^{5}$ In the iodite-arsenous acid system the shape of the convective front varies as the width of the tube is increased. However, for every tube there is a single, unique front formed. This is strikingly different from the theory in porous media presented here, where we can have a region of bistability. This bistability was not observed in the experiments in vertical tubes, nor in calculations using the Navier-Stokes equation. ${ }^{14}$ The viscous flow provides a region where only the nonsymmetric state is stable. The shape of the front is symmetric for large diameters which coincides with our calculations. The lack of bistability in the viscous flow may be due to the boundary conditions. Free boundary conditions in viscous fluids have not been investigated yet.
To compare our theory with experiments in a HeleShaw cell will require a very small separation between the walls. For example, for a wall separation of $0.05 \mathrm{~cm}$, the corresponding critical wavelength for onset of convection is about $0.03 \mathrm{~cm}$ in an aqueous solution. To test the critical wavelength requires a reduction the Rayleigh number which in turn increases the critical wavelength. This can be achieved by controlling the density difference or the viscosity of the solution. Once this is achieved, the onset of convection can be studied by changing the wall separation. Recent experiments by Carey et al. observed fingering in the iodate-arsenous reaction in a Hele-Shaw cell. ${ }^{15}$ These experiments are away from the onset of convection while the present calculations are only valid near onset. New experiments may be designed to study the onset of convection.

\section{ACKNOWLEDGMENTS}

J. W. Wilder and B. F. Edwards acknowledge support from NSF Grant Number RII-8922106. D. A. Vasquez acknowledges support from Research Corporation and the Purdue University Research Fund. The authors thank Yunqing $\mathrm{Wu}$ for helpful discussions. We thank M. R. Carey, S. W. Morris, and P. K. Kolodner for communicating their experimental results prior to publication.

${ }^{1}$ Oscillations and Traveling Waves in Chemical Systems, edited by R. J. Field and M. Burger (Wiley, New York, 1985).

${ }^{2}$ J. A. Pojman and I. R. Epstein, J. Phys. Chem. 94, 4966 (1990).

${ }^{3}$ T. McManus, Ph.D. thesis, West Virginia University, 1989; J. A. Pojman, I. R. Epstein, T. J. McManus, and K. Showalter, J. Phys. Chem. 95, 1299 (1991).

${ }^{4}$ G. Bazsa and I. R. Epstein, J. Phys. Chem. 89, 3050 (1985), Item 5; J. Masere, D. A. Vasquez, B. F. Edwards, J. W. Wilder, and K. Showalter, J. Phys. Chem. 26, 6505 (1994)

${ }^{5}$ P. G. Saffman and G. Taylor, Proc. R. Soc. London Ser. A 245, 312 (1958)

${ }^{6}$ J. W. Wilder, D. A. Vasquez, and B. F. Edwards, Phys. Rev. E 47, 3761 (1993).

${ }^{7}$ A. Saul and K. Showalter, in Oscillations and Traveling Waves in Chemical Systems, edited by R. J. Field and M. Burger (Wiley, New York, 1985), p. 419.

${ }^{8}$ D. A. Vasquez, J. W. Wilder, and B. F. Edwards, J. Chem. Phys. 98, 2138 (1993).

${ }^{9}$ J. Huang, D. A. Vasquez, B. F. Edwards, and P. Kolodner, Phys. Rev. E 48, 4378 (1993).

${ }^{10}$ R. Sweet, SIAM (Soc. INd. Appl. Math.) J. Numer. Anal. 14, 706 (1977); J. Adams, P. Swarztrauber, and R. Seet, FISHPACK (The National Center for Atmospheric Research, Boulder, 1980).

${ }^{11}$ I. P. Nagy, A. Keresztessy, and J. A. Pojman, J. Phys. Chem. 99, 5385 (1995)

${ }^{12}$ H. Miike, H. Yamamoto, S. Kai, and S. C. Muller, Phys. Rev. E 48, 1627 (1993).

${ }^{13}$ Y. Wu, D. A. Vasquez, J. W. Wilder, and B. F. Edwards, Phys. Rev. E (to be published).

${ }^{14}$ M. R. Carey, S. W. Morris, and P. K. Kolodner (unpublished). 